TRANSACTIONS OF THE

AMERICAN MATHEMATICAL SOCIETY

Volume 315, Number 2, October 1989

\title{
ON A MAXIMAL FUNCTION ON COMPACT LIE GROUPS
}

\author{
MICHAEL COWLING AND CHRISTOPHER MEANEY
}

\begin{abstract}
Suppose that $G$ is a compact Lie group with finite centre. For each positive number $s$ we consider the $\operatorname{Ad}(G)$-invariant probability measure $\mu_{s}$ carried on the conjugacy class of $\exp \left(s H_{\rho}\right)$ in $G$. This one-parameter family of measures is used to define a maximal function $\mathscr{M} f$, for each continuous function $f$ on $G$. Our theorem states that there is an index $p_{0}$ in $(1,2)$, depending on $G$, such that the maximal operator $\mathscr{M}$ is bounded on $L^{p}(G)$ when $p$ is greater than $p_{0}$. When the rank of $G$ is greater than one, this provides an example of a controllable maximal operator coming from averages over a family of submanifolds, each of codimension greater than one.
\end{abstract}

\section{INTRODUCTION}

Let $G$ be a compact Lie group of rank $r$ with finite centre, with its Haar measure normalized to have total mass 1 . In $G$ we pick a maximal torus $T$, and denote the corresponding Lie algebras by $\mathfrak{g}$ and $t$. In $\mathfrak{t}_{C}^{*}$ we fix a system of positive roots, $\Phi^{+}$say, and the special element

$$
\rho=\frac{1}{2} \sum_{\alpha \in \Phi^{+}} \alpha .
$$

Let $\left\{\alpha_{1}, \ldots, \alpha_{r}\right\}$ denote the set of simple positive roots. Each positive root is an integral combination of the simple roots-

$$
\alpha=\sum_{j=1}^{r} c_{j}(\alpha) \alpha_{j},
$$

say, where $c_{j}(\alpha) \geq 0$. We will need a lower bound on the number of positive roots $\alpha$ for which $c_{j}(\alpha) \geq 1$. Define the ( $G$-dependent) constant $\gamma$ by the rule

$$
\gamma=\min _{1 \leq j \leq r}\left|\left\{\alpha \in \Phi^{+}: c_{j}(\alpha) \geq 1\right\}\right|
$$

Clearly, for each $J$ in $\{1, \ldots, r\}$, the number of positive roots $\alpha$ for which $c_{J}(\alpha)>0$ is at least $\gamma$.

The Lie algebra $\mathfrak{g}$ is equipped with the positive definite inner product coming from the Killing form, and for each $\mu \in i \mathrm{t}^{*}$ we define $H_{\mu} \in \mathfrak{t}$ so that

$$
\mu(X)=i\left(H_{\mu} \mid X\right) \quad \forall X \in \mathfrak{t} .
$$

Received by the editors April 15, 1988.

1980 Mathematics Subject Classification (1985 Revision). Primary 42B25; 22E30; 43A75. 
In addition, we denote by $|\cdot|$ the norm on $t$ and $t^{*}$ induced by this inner product. Fix a positive number $R$ so that $\exp \left(s H_{\rho}\right)$ is regular in $T$ when $0<s<R$. It is known that $G$ is the union of the conjugacy classes of the elements of $T$ and that the conjugacy class of a regular element of $T$ is a closed submanifold whose codimension is equal to the rank $r$ of $G$. We now concentrate on the conjugacy classes of the elements $\exp \left(s H_{\rho}\right)$ in $G$. For each such $s$, let $\mu_{s}$ denote the $\operatorname{Ad}(G)$-invariant probability measure carried on the conjugacy class of $\exp \left(s H_{\rho}\right)$, defined by the formula

$$
\int_{G} f d \mu_{s}=\int_{G} f\left(g \exp \left(s H_{\rho}\right) g^{-1}\right) d t \quad \forall f \in C(G) .
$$

We can use convolution with these measures to form a one parameter family of mean-value operators. That is, for any $f$ in $C(G)$ and $x$ in $G$, set

$$
f * \mu_{s}(x)=\int_{G} f\left(x g \exp \left(s H_{\rho}\right) g^{-1}\right) d g .
$$

This is analogous to the spherical mean values of a function on Euclidean space, but now arises from the averages of the function over submanifolds of codimension $r$.

For each continuous function $f$ on $G$, we consider the maximal function $\mathscr{M} f-$

$$
\mathscr{M} f(x)=\sup _{s \in(0, R)}\left|f * \mu_{s}(x)\right| \quad \forall x \in G .
$$

This is suggested by the results on spherical mean values on Euclidean spaces described by E. M. Stein in [St2]. In that paper he showed that his maximal operators are bounded on $L^{p}\left(\mathbf{R}^{n}\right)$ when $p>n /(n-1)$ and $n \geq 3$. Here we prove the following a priori estimates, which extend, as in Stein's work, to all $L^{p}(G)$-functions.

Theorem. Let $G$ and $\gamma$ be as above. Then, if $p>1+[2 \gamma]^{-1}$,

$$
\|\mathscr{M} f\|_{p} \leq C_{p}\|f\|_{p} \quad \forall f \in C(G) .
$$

As in Stein's work, the keys to the proof are $g$-function techniques, and decay estimates on the Fourier transform of $\mu_{s}$. M. Christ [Ch] has recently obtained related results. He considers averages over families of submanifolds of Euclidean space subject to certain rotational curvature conditions. In the same vein there is the recent work of C. D. Sogge and Stein [SS].

Our paper is organised as follows. We begin by recalling a few facts from the theory of compact Lie groups and we describe the role of $\gamma$ in the proof. Next, we derive some estimates on the Fourier transform of $\mu_{s}$ (Lemma 1) which we use to control some more singular variants of $\mu_{s}$ (Lemma 2). Then the proof of the theorem is fairly straightforward. We conclude with an appendix calculating $\gamma$ for an arbitrary compact Lie group $G$.

Throughout this paper, $C$ denotes a constant (possibly depending on $G$ ), which may vary from line to line. 


\section{LIE THEORETIC DETAILS}

Let $\widehat{G}$, the dual object of $G$, be parametrized by $\Lambda$, the appropriate sublattice of the lattice of dominant integral weights with respect to $\Phi^{+}$. To each dominant integral weight $\lambda$ in $\Lambda$ we associate the representation $\pi_{\lambda}$, the character $\chi_{\lambda}$ and the dimension $d_{\lambda}$, equal to $\chi_{\lambda}(1)$. The measures $\mu_{s}$ are central on $G$ and their Fourier series on $G$ will consist of sums of Fourier coefficients multiplied by characters, namely,

$$
\sum_{\lambda \in \Lambda} \chi_{\lambda}\left(\exp \left(s H_{\rho}\right)\right) \chi_{\lambda}(x)=\sum_{\lambda \in \Lambda} d_{\lambda} \hat{\mu}_{s}(\lambda) \chi_{\lambda}(x) \quad \forall x \in G .
$$

In estimating $\hat{\mu}_{s}(\lambda)$ and its derivatives with respect to $s$, we will need to know the relationship between $|\lambda|$ and the terms $(\lambda+\rho \mid \alpha)$ as $\alpha$ varies over the set of positive roots.

First, since $\left\{\alpha_{1}, \ldots, \alpha_{r}\right\}$ is a basis of $\mathfrak{t}_{\mathrm{C}}^{*}$, there is a constant $C$ such that

$$
\max _{j=1, \ldots, r}\left|\left(\lambda \mid \alpha_{j}\right)\right| \geq C|\lambda| \quad \forall \lambda \in \mathfrak{t}_{\mathbf{C}}^{*} \text {. }
$$

Next, given a dominant weight $\lambda$, choose $J$ in $\{1, \ldots, r\}$ such that $\left(\lambda+\rho \mid \alpha_{J}\right)$ is maximal. From (1),

$$
(\lambda+\rho \mid \alpha)=\sum_{j=1}^{r} c_{j}(\alpha)\left(\lambda+\rho \mid \alpha_{j}\right) \geq c_{J}(\alpha)\left(\lambda+\rho \mid \alpha_{J}\right) \geq C c_{J}(\alpha)|\lambda+\rho| .
$$

There are at least $\gamma$ positive roots $\alpha$ for which $c_{J}(\alpha) \geq 1$, and for the remaining $\alpha$ in $\Phi^{+},(\lambda+\rho \mid \alpha) \geq(\rho \mid \alpha) \geq C>0$. Consequently there is another constant $C$ such that

$$
\prod_{\alpha \in \Phi^{+}}(\lambda+\rho \mid \alpha) \geq C|\lambda+\rho|^{\gamma} \quad \forall \lambda \in \Lambda .
$$

The other Lie theoretic result we shall need is the following consequence of the Weyl character formula (see page 106 of [Wa] for the details):

$$
\hat{\mu}_{s}(\lambda)=\prod_{\alpha \in \Phi^{+}} \frac{\sin (s(\lambda+\rho \mid \alpha)) s(\rho \mid \alpha)}{s(\lambda+\rho \mid \alpha) \sin (s(\rho \mid \alpha))} \quad \forall \lambda \in \Lambda, \forall s \in(0, R) .
$$

\section{FOURIER TRANSFORM ESTIMATES}

Here we estimate the Fourier transform of $\mu_{s}$ and of some derived distributions.

Lemma 1. For each $k$ in $\{0,1,2, \ldots\}$, there exist positive constants $C_{k}$ such that, for all $\lambda$ in $\Lambda, t$ in $(0,1)$ and $s$ in $(0, R)$,

$$
\left|\left(\frac{\partial}{\partial t}\right)^{k}\left(\hat{\mu}_{s t}(\lambda)\right)\right| \leq C_{k} s^{k} \frac{(1+|\lambda|)^{k}}{(1+s t|\lambda|)^{\gamma}}
$$


and

(b)

$$
\left|\left(\frac{\partial}{\partial t}\right)^{k}\left(\hat{\mu}_{s t}(\lambda)-1\right)\right| \leq C_{k} s|\lambda| .
$$

Proof. Both estimates use formula (3). Inequality (a) follows from Leibnitz' rule for the derivatives of products and the easy estimates

$$
\left|\left(\frac{\partial}{\partial t}\right)^{m} \frac{\sin (t(\lambda+\rho \mid \alpha))}{t(\lambda+\rho \mid \alpha)}\right| \leq C_{\alpha, m} \frac{(1+|\lambda|)^{m}}{1+t(\lambda+\rho \mid \alpha)}
$$

and

$$
\left|\left(\frac{\partial}{\partial t}\right)^{m} \frac{t(\rho \mid \alpha)}{\sin (t(\rho \mid \alpha))}\right| \leq C_{\alpha, m}
$$

for the appropriate constants $C_{\alpha, m}$, valid when $t$ is in $[0,1]$ and $s$ is in $[0, R]$. The product in (3) is taken over all positive roots and in particular, from (2),

$$
\prod_{\alpha \in \Phi^{+}} \frac{1}{1+t(\lambda+\rho \mid \alpha)} \leq \frac{C}{(1+t|\lambda|)^{\gamma}} .
$$

To prove (b), if $\lambda=0$, there is nothing to do, as $\hat{\mu}_{s}(0)=1$. Otherwise, we take the Maclaurin expansions of

$$
\frac{\sin (s(\lambda+\rho \mid \alpha))}{s(\lambda+\rho \mid \alpha)} \text { and } \frac{s(\rho \mid \alpha)}{\sin (s(\rho \mid \alpha))},
$$

convergent for small $s$, and multiply together the results.

Following [CM], we define $R_{a, z} \mu_{s}$ and $R_{b, w} R_{a, z} \mu_{s}$. When $\operatorname{Re}(a)$ and $\operatorname{Re}(z)$ are positive, and $u$ is in $C^{\infty}(G)$, we set

$$
\begin{aligned}
\left(R_{a, z} \mu_{s}\right)(u) & =2 \Gamma(z)^{-1} \int_{0}^{1} t^{a-1}\left(1-t^{2}\right)^{z-1} \mu_{s t}(u) d t \\
& =2 \Gamma(z)^{-1} \int_{G} \int_{0}^{1} t^{a-1}\left(1-t^{2}\right)^{z-1} u\left(\exp \left(\operatorname{st} \operatorname{Ad}(g) H_{\rho}\right)\right) d t d g .
\end{aligned}
$$

The $\mathbf{R}^{n}$ theory can be easily modified to show that this continues analytically in $z$ to an entire function, and hence $R_{a, z} \mu_{s}$ is defined as a distribution on $G$. Analogously, when $\operatorname{Re}(a), \operatorname{Re}(b), \operatorname{Re}(z)$ and $\operatorname{Re}(w)$ are all positive, we define $\left(R_{b, w} R_{a, z} \mu_{s}\right)(u)$ to be

$$
\begin{aligned}
\frac{4}{\Gamma(z) \Gamma(w)} \int_{G} \int_{0}^{1} \int_{0}^{1} t_{1}^{a-1}\left(1-t_{1}^{2}\right)^{z-1} t_{2}^{b-1} & \left(1-t_{2}^{2}\right)^{w-1} \\
& \times u\left(\exp \left(s t_{1} t_{2} \operatorname{Ad}(g) H_{\rho}\right)\right) d t_{1} d t_{2} d g .
\end{aligned}
$$

By expanding the function $\left(t_{1}, t_{2}\right) \mapsto u\left(\exp \left(s t_{1} t_{2} \operatorname{Ad}(g) H_{p}\right)\right)$ in a Taylor series about $(1,1)$, it is easy to check that this expression continues analytically in $z$ and $w$; this procedure defines $R_{b, w} R_{a, z} \mu_{s}$ when $\operatorname{Re}(z) \leq 0$ or $\operatorname{Re}(w) \leq 0$. 
Following [CM, Lemma 1.2], it is easy to verify that if $w \in\left[-\frac{3}{4},-\frac{1}{2}\right]$, and $b \geq 2$, then for all $u$ in $C^{\infty}(G)$,

$$
\left(R_{a, z} \mu_{s}\right)(u)=2 \Gamma(-w)^{-1} \int_{0}^{1} t^{b+2 w-1}\left(1-t^{2}\right)^{-w-1} R_{b, w} R_{a, z} \mu_{s t}(u) d t
$$

Lemma 2. If $\varepsilon$ is positive, $\operatorname{Re}(z)$ is in $(-\gamma, 0), \operatorname{Re}(w)$ is in $(-1,0), \operatorname{Re}(z)+$ $\operatorname{Re}(w)>-\gamma, \operatorname{Re}(a)>2 \gamma$, and $\operatorname{Re}(b)>\gamma+1$, then for all $s$ in $[0, R]$ and $\lambda$ in $\Lambda$,

$$
\begin{gathered}
\left|\left(R_{b, s} R_{a, z} \mu_{s}\right)^{-}(\lambda)\right| \leq C_{a, b, w, z, \varepsilon}(1+s|\lambda|)^{-\gamma-\operatorname{Re}(z)-\operatorname{Re}(w)+\varepsilon}, \\
\left|\left(R_{b, w} R_{a, z} \mu_{s}\right)^{-}(\lambda)-\frac{\Gamma(a / 2) \Gamma(b / 2)}{\Gamma(a / 2+z) \Gamma(b / 2+w)}\right| \leq C_{z, w} s|\lambda|, \\
\left|\left(R_{2,1} \mu_{s}\right)^{-}(\lambda)\right| \leq C(1+s|\lambda|)^{-\gamma}, \\
\left|\left(R_{2,1} \mu_{s}\right)^{-}(\lambda)-1\right| \leq C s|\lambda| .
\end{gathered}
$$

In (a) and (b), the constants grow at most exponentially in $\operatorname{Im}(z)$ and $\operatorname{Im}(w)$, provided that $\operatorname{Re}(z)$ and $\operatorname{Re}(w)$ vary over compact subsets of $(-\gamma, 0)$ and $(-1,0)$.

Proof. It suffices to deal with the case $\lambda \neq 0$, so we may assume that $|\lambda| \geq C>$ 0 .

Proof of (a). Choose $\delta$ greater than 0. We first show that if $\operatorname{Re}(z)=\operatorname{Re}(w)=\delta$, then

$$
\left|\left(R_{b, w} R_{a, z} \mu_{s}\right)^{-}(\lambda)\right| \leq C_{z, w}(1+s|\lambda|)^{-\gamma},
$$

and next, if $\operatorname{Re}(z)=\delta$ and $\operatorname{Re}(w)=\delta-1$, then

$$
\left|\left(R_{b, w} R_{a, z} \mu_{s}\right)^{-}(\lambda)\right| \leq C_{z, w}(1+s|\lambda|)^{1-\gamma} .
$$

We also show that if $\operatorname{Re}(z)=\delta-\gamma$ and $\operatorname{Re}(w)=\delta$, then

$$
\left|\left(R_{b, w} R_{a, z} \mu_{s}\right)^{\wedge}(\lambda)\right| \leq C_{z, w},
$$

and deduce similarly that if $\operatorname{Re}(z)=\delta-\gamma+1$ and $\operatorname{Re}(w)=\delta-1$, then

$$
\left|\left(R_{b, w} R_{a, z} \mu_{s}\right)^{\wedge}(\lambda)\right| \leq C_{z, w} .
$$

By judicious application of the three lines theorem, (a) follows.

To prove (5), we note that

$$
\begin{aligned}
& \left|\left(R_{b, w} R_{a, z} \mu_{s}\right)^{-}(\lambda)\right| \\
& \quad=\left|\frac{4}{\Gamma(z) \Gamma(w)} \int_{0}^{1} \int_{0}^{1} t_{1}^{a-1}\left(1-t_{1}^{2}\right)^{z-1} t_{2}^{b-1}\left(1-t_{2}^{2}\right)^{w-1} \hat{\mu}_{s t_{1} t_{2}}(\lambda) d t_{1} d t_{2}\right| \\
& \quad \leq \frac{4}{|\Gamma(z) \Gamma(w)|} \int_{0}^{1} \int_{0}^{1}\left|t_{1}^{a-1} t_{2}^{b-1}\right|\left(1-t_{1}^{2}\right)^{\delta-1}\left(1-t_{2}^{2}\right)^{\delta-1}\left|\hat{\mu}_{s t_{1} t_{2}}(\lambda)\right| d t_{1} d t_{2} .
\end{aligned}
$$


This double integral is estimated by splitting into two parts: in the first, when $s t_{1} t_{2}|\lambda| \leq 1$, we use the estimates $\left(t_{1} t_{2}\right)^{\gamma} \leq(s|\lambda|)^{-\gamma}$ and $\left|\hat{\mu}_{s t_{1} t_{2}}(\lambda)\right| \leq 1$, and in the second, when $s t_{1} t_{2}|\lambda|>1$, we use the estimate (Lemma $1(\mathrm{a})$ )

$$
\left|\hat{\mu}_{s t_{1} t_{2}}(\lambda)\right| \leq C\left(s t_{1} t_{2}|\lambda|\right)^{-\gamma} \text {. }
$$

Consequently

$$
\begin{aligned}
& \left|\left(R_{b, w} R_{a, z} \mu_{s}\right)^{\wedge}(\lambda)\right| \\
& \quad \leq \frac{4(1+C)}{|\Gamma(w) \Gamma(z)|}\left\{\int_{0}^{1} \int_{0}^{1}\left|t_{1}^{a-\gamma-1} t_{2}^{b-\gamma-1}\right|\left(1-t_{1}^{2}\right)^{\delta-1}\left(1-t_{2}^{2}\right)^{\delta-1} d t_{1} d t_{2}\right\}(s|\lambda|)^{-\gamma},
\end{aligned}
$$

and (5) follows.

Next, to prove (6), we integrate by parts to obtain the formula, initially valid when $\operatorname{Re}(w)$ and $\operatorname{Re}(z)>0$, but by analytic continuation also when $\operatorname{Re}(z)>0$ and $\operatorname{Re}(w)>-1$,

$$
\begin{aligned}
\left(R_{b, w} R_{a, z} \mu_{s}\right)^{-}(\lambda) & \\
= & \frac{2}{w \Gamma(w) \Gamma(z)} \int_{0}^{1} \int_{0}^{1} t_{1}^{a-1}\left(1-t_{1}^{2}\right)^{z-1} 2 w t_{2}\left(1-t_{2}^{2}\right)^{w-1} t_{2}^{b-2} \hat{\mu}_{s t_{1} t_{2}}(\lambda) d t_{1} d t_{2} \\
= & \frac{-2}{\Gamma(w+1) \Gamma(z)} \int_{0}^{1} \int_{0}^{1} t_{1}^{a-1}\left(1-t_{1}^{2}\right)^{z-1}\left[\frac{\partial}{\partial t_{2}}\left(1-t_{2}^{2}\right) w\right] t_{2}^{b-2} \hat{\mu}_{s t_{1} t_{2}}(\lambda) d t_{1} d t_{2} \\
= & \frac{2(b-2)}{\Gamma(w+1) \Gamma(z)} \int_{0}^{1} \int_{0}^{1} t_{1}^{a-1}\left(1-t_{1}^{2}\right)^{z-1}\left(1-t_{2}^{2}\right)^{w} t_{2}^{b-3} \hat{\mu}_{s t_{1} t_{2}}(\lambda) d t_{1} d t_{2} \\
& +\frac{2}{\Gamma(w+1) \Gamma(z)} \int_{0}^{1} \int_{0}^{1} t_{1}^{a-1}\left(1-t_{1}^{2}\right)^{z-1}\left(1-t_{2}^{2}\right)^{w} t_{2}^{b-2}\left[\frac{\partial}{\partial t_{2}} \hat{\mu}_{s t_{1} t_{2}}(\lambda)\right] d t_{1} d t_{2} .
\end{aligned}
$$

It follows that, when $\operatorname{Re}(z)=\delta$ and $\operatorname{Re}(w)=\delta-1$,

$$
\begin{aligned}
& \left|\left(R_{b, w} R_{a, z} \mu_{s}\right)^{-}(\lambda)\right| \\
& \leq \frac{2|b-2|}{|\Gamma(w+1) \Gamma(z)|} \int_{0}^{1} \int_{0}^{1}\left|t_{1}^{a-1} t_{2}^{b-3}\right|\left(1-t_{1}^{2}\right)^{\delta-1}\left(1-t_{2}^{2}\right)^{\delta-1}\left|\hat{\mu}_{s t_{1} t_{2}}(\lambda)\right| d t_{1} d t_{2} \\
& \quad+\frac{2}{|\Gamma(w+1) \Gamma(z)|} \int_{0}^{1} \int_{0}^{1}\left|t_{1}^{a-1} t_{2}^{b-2}\right| \\
& \cdot\left(1-t_{1}^{2}\right)^{\delta-1}\left(1-t_{2}^{2}\right)^{\delta-1}\left|\frac{\partial}{\partial t_{2}} \hat{\mu}_{s t_{1} t_{2}}(\lambda)\right| d t_{1} d t_{2} .
\end{aligned}
$$

We split each of the two integrals into two parts, and use Lemma 1. For the first integral, when $s t_{1} t_{2}|\lambda| \leq 1$, we use the estimates $\left(t_{1} t_{2}\right)^{\gamma-1} \leq(s|\lambda|)^{1-\gamma}$ and $\left|\hat{\mu}_{s t_{1} t_{2}}(\lambda)\right| \leq 1$, while if $s t_{1} t_{2}|\lambda|>1$, we use the estimate

$$
\left|\hat{\mu}_{s t_{1} t_{2}}(\lambda)\right| \leq C\left(s t_{1} t_{2}|\lambda|\right)^{1-\gamma} ;
$$

for the second integral, when $s t_{1} t_{2}|\lambda| \leq 1$, we use the estimates $\left(t_{1} t_{2}\right)^{\gamma} \leq(s|\lambda|)^{-\gamma}$ and

$$
\left|\frac{\partial}{\partial t_{2}} \hat{\mu}_{s t_{1} t_{2}}(\lambda)\right| \leq C s t_{1}|\lambda|
$$


while if $s t_{1} t_{2}|\lambda|>1$, we use the estimate

$$
\left|\frac{\partial}{\partial t_{2}} \hat{\mu}_{s t_{1} t_{2}}(\lambda)\right| \leq C s t_{1}|\lambda|\left(s t_{1} t_{2}|\lambda|\right)^{-\gamma} \text {. }
$$

It follows that

$$
\begin{aligned}
& \left|\left(R_{b, w} R_{a, z} \mu_{s}\right)^{-}(\lambda)\right| \\
& \quad \leq C\left\{\int_{0}^{1} \int_{0}^{1}\left|t_{1}^{a-\gamma} t_{2}^{b-\gamma-2}\right|\left(1-t_{1}^{2}\right)^{\delta-1}\left(1-t_{2}^{2}\right)^{\delta-1} d t_{1} d t_{2}\right\}(s|\lambda|)^{1-\gamma},
\end{aligned}
$$

proving (6).

The idea of the proof of (7) is similar. Let $K$ be a positive integer. By integrating by parts $K$ times, we obtain the formula, valid when $\operatorname{Re}(w)=\delta$ and $\operatorname{Re}(z)=\delta-K$,

$$
\begin{aligned}
& \left(R_{b, w} R_{a, z} \mu_{s}\right)^{\wedge}(\lambda) \\
& \quad=\frac{2^{2-K}}{\Gamma(w) \Gamma(z+K)} \int_{0}^{1} \int_{0}^{1} t_{2}^{b-1}\left(1-t_{2}^{2}\right)^{w-1}\left(1-t_{1}^{2}\right)^{z+K-1} F_{K}\left(s, t_{1}, t_{2}, \lambda\right) d t_{1} d t_{2},
\end{aligned}
$$

where

$$
F_{K}\left(s, t_{1}, t_{2}, \lambda\right)=\frac{\partial}{\partial t_{1}} t_{1}^{-1} \cdots \frac{\partial}{\partial t_{1}} t_{1}^{-1} \frac{\partial}{\partial t_{1}} t_{1}^{a-2} \hat{\mu}_{s t_{1} t_{2}}(\lambda),
$$

there being a total of $K$ derivatives. Now, for appropriate coefficients $c(a, k, K)$,

$$
F_{K}\left(s, t_{1}, t_{2}, \lambda\right)=\sum_{k=0}^{K} c(a, k, K) t_{1}^{a+k-2 K-1}\left(\frac{\partial}{\partial t_{1}}\right)^{k} \hat{\mu}_{s t_{1} t_{2}}(\lambda),
$$

and the same type of estimation as before proves (7). Indeed,

$$
\begin{aligned}
& \left|\left(R_{b, w} R_{a, z} \mu_{s}\right)^{\wedge}(\lambda)\right| \\
& \leq \sum_{k=0}^{K} \frac{2^{2-K}|c(a, k, K)|}{|\Gamma(w) \Gamma(z+K)|} \int_{0}^{1} \int_{0}^{1}\left|t_{1}^{a+k-2 K-1} t_{2}^{b-1}\right| \\
& \cdot\left(1-t_{1}^{2}\right)^{\delta-1}\left(1-t_{2}^{2}\right)^{\delta-1}\left|\left(\frac{\partial}{\partial t_{1}}\right)^{k} \hat{\mu}_{s t_{1} t_{2}}(\lambda)\right| d t_{1} d t_{2} .
\end{aligned}
$$

In these integrals, we use the estimates

$$
\left(t_{1} t_{2}\right)^{k+\gamma-K} \leq(s|\lambda|)^{K-k-\gamma} \text { and }\left|\left(\partial / \partial t_{1}\right)^{k} \hat{\mu}_{s t_{1} t_{2}}(\lambda)\right| \leq C\left(s t_{2}|\lambda|\right)^{k}
$$

when $s t_{1} t_{2}|\lambda| \leq 1$, while if $s t_{1} t_{2}|\lambda|>1$, we use the estimate

$$
\left|\left(\frac{\partial}{\partial t_{1}}\right)^{k} \hat{\mu}_{s t_{1} t_{2}}(\lambda)\right| \leq C\left(s t_{2}|\lambda|\right)^{k}\left(s t_{1} t_{2}|\lambda|\right)^{K-k-\gamma} \text {. }
$$

As before, it follows that

$$
\left|\left(R_{b, w} R_{a, z} \mu_{s}\right)^{\wedge}(\lambda)\right| \leq C_{z, w}(1+s|\lambda|)^{K-\gamma},
$$

provided that $K \leq \gamma$, proving (7). 
The proof of (8) is very similar. We integrate by parts $\gamma-1$ times in $t_{1}$, and once in $t_{2}$, and obtain the formula, valid when $\operatorname{Re}(z)=\delta+1-\gamma$ and $\operatorname{Re}(w)=\delta-1$,

$$
\begin{aligned}
\Gamma(w+1) \Gamma(z+\gamma-1)\left(R_{b, w} R_{a, z} \mu_{s}\right)^{-}(\lambda) \\
\quad=2^{2-\gamma} \int_{0}^{1} \int_{0}^{1}\left(1-t_{1}^{2}\right)^{z+\gamma-2}\left(1-t_{2}^{2}\right)^{w} \frac{\partial}{\partial t_{2}}\left[t_{2}^{b-2} F_{\gamma-1}\left(s_{t}, t_{1}, t_{2}, \lambda\right)\right] d t_{1} d t_{2},
\end{aligned}
$$

and the same type of estimate as for (7) also proves (8).

Proof of (b). As in the proof of (a), when $\operatorname{Re}(z)+\gamma>0$ and $\operatorname{Re}(w)>-1$,

$$
\begin{aligned}
\Gamma(w+1) \Gamma(z+\gamma)\left(R_{b, w} R_{a, z} \mu_{s}\right) & (\lambda) \\
& =2^{1-\gamma} \int_{0}^{1} \int_{0}^{1}\left(1-t_{1}^{2}\right)^{z+\gamma-1}\left(1-t_{2}^{2}\right)^{w} \frac{\partial}{\partial t_{2}}\left\{t_{2}^{b-2} F_{\gamma}\left(s, t_{1}, t_{2}, \lambda\right)\right\} d t_{1} d t_{2} ;
\end{aligned}
$$

this implies that

$$
\begin{aligned}
\left(R_{b, w}\right. & \left.R_{a, z} \mu_{0}\right) \hat{(\lambda)} \\
& =\frac{2^{1-\gamma}}{\Gamma(w+1) \Gamma(z+\gamma)} \int_{0}^{1} \int_{0}^{1}\left(1-t_{1}^{2}\right)^{z+\gamma-1}\left(1-t_{2}^{2}\right)^{w}(b-2) t_{2}^{b-3} \\
& =\frac{\Gamma(a / 2) \Gamma(b / 2)}{\Gamma(a / 2+z) \Gamma(b / 2+w)},
\end{aligned}
$$

and that

$$
\begin{aligned}
& \left|\left(R_{b, w} R_{a, z} \mu_{s}\right)^{-}(\lambda)-\frac{\Gamma(a / 2) \Gamma(b / 2)}{\Gamma(a / 2+z) \Gamma(b / 2+z)}\right| \\
& \leq \frac{2^{1-\gamma}}{|\Gamma(w+1) \Gamma(z+\gamma)|} \int_{0}^{1} \int_{0}^{1}\left|\left(1-t_{1}^{2}\right)^{z+\gamma-1}\left(1-t_{2}^{2}\right)^{w}\right| \\
& \cdot\left|\left(\frac{\partial}{\partial t_{2}}\right) t_{2}^{b-2}\left(\frac{\partial}{\partial t_{1}}\right) t_{1}^{-1} \cdots\left(\frac{\partial}{\partial t_{1}}\right) t_{1}^{a-2}\left(\hat{\mu}_{s t_{1} t_{2}}(\lambda)-1\right)\right| d t_{1} d t_{2} .
\end{aligned}
$$

To estimate this integral, we write out the second part of the integrand:

$$
\begin{aligned}
\left(\frac{\partial}{\partial t_{2}}\right) & t_{2}^{b-2}\left(\frac{\partial}{\partial t_{1}}\right) t_{1}^{-1} \cdots\left(\frac{\partial}{\partial t_{1}}\right) t_{1}^{a-2}\left(\hat{\mu}_{s t_{1} t_{2}}(\lambda)-1\right) \\
= & (b-2) \sum_{k=0}^{\gamma} c(a, k, \gamma) t_{1}^{a+k-1-2 \gamma} t_{2}^{b-3}\left(\frac{\partial}{\partial t_{1}}\right)^{k}\left(\hat{\mu}_{s t_{1} t_{2}}(\lambda)-1\right) \\
& +\sum_{k=0}^{\gamma} c(a, k, \gamma) t_{1}^{a+k-1-2 \gamma} t_{2}^{b-2}\left(\frac{\partial}{\partial t_{2}}\right)\left(\frac{\partial}{\partial t_{1}}\right)^{k}\left(\hat{\mu}_{s t_{1} t_{2}}(\lambda)-1\right) .
\end{aligned}
$$

By using the estimates

$$
\left|\left(\frac{\partial}{\partial t_{1}}\right)^{k}\left\{\hat{\mu}_{s t_{1} t_{2}}(\lambda)-1\right\}\right| \leq C s|\lambda|
$$


and

$$
\left|\left(\frac{\partial}{\partial t_{2}}\right)\left(\frac{\partial}{\partial t_{1}}\right)^{k}\left\{\hat{\mu}_{s t_{1} t_{2}}(\lambda)-1\right\}\right| \leq C s|\lambda|
$$

from Lemma $1(\mathrm{~b})$, and the preceding equation and estimate, it is easy to obtain the desired result.

The proofs of (c) and (d) are easy variants of the above proofs.

\section{THE PROOF OF THE THEOREM}

The idea of the proof is to subtract a better behaved maximal operator, which is certainly bounded on $L^{p}(G)$, from the maximal operator being considered. The difference is controlled by $g$-function techniques, which are developments of Tauberian methods. As this does not quite yield the optimal result, the same trick is actually applied to a more singular operator, acting on $L^{2}(G)$; as a less singular operator is bounded on all $L^{p}(G) \quad(1<p<\infty)$, interpolation techniques give the sharper result stated.

First we find the better behaved maximal operator described above.

Lemma 3. Suppose that $\operatorname{Re}(z) \geq 1$. Then for any $p$ in $(1, \infty)$,

$$
\left\|\sup _{s \in(0, R)}\left|\left(R_{a, z} \mu_{s}\right) * f\right|\right\|_{p} \leq C_{z}\|f\|_{p} \quad \forall f \in C(G) .
$$

Proof. This is best seen by the transference methods of Coifman and Weiss [CW]. For fixed $g$ in $G$, let $\lambda_{s}$ be the measure supported by the one-parameter subgroup $\exp \left(\operatorname{RAd}(g) H_{\rho}\right)$, given by

$$
\lambda_{s}(f)=\frac{2}{\Gamma(z)} \int_{0}^{1} t^{a-1}\left(1-t^{2}\right)^{z-1} f\left(\exp \left(s t \operatorname{Ad}(g) H_{\rho}\right)\right) d t \quad \forall f \in C(G) .
$$

Coifman and Weiss show that, if $1<p \leq \infty$, then

$$
\left\|\sup _{s \in(0, R)}\left|\lambda_{s} * f\right|\right\|_{p} \leq C_{p}\|f\|_{p} \quad \forall f \in C(G) .
$$

By integrating the various $\lambda_{s}$ as $g$ varies, we obtain the lemma.

Now we fix $a$, bigger than $2 \gamma$. The main step of the proof of the theorem is to show that, if $z$ satisfies $\operatorname{Re}(z)+\gamma>\frac{1}{2}$, then

$$
\left\|\sup _{s \in(0, R)}\left|\left(R_{a, z} \mu_{s}\right) * f\right|\right\|_{2} \leq C_{z}\|f\|_{2} \quad \forall f \in C(G) .
$$

Assuming this for the moment, we recall that, if $\operatorname{Re}(z) \geq 1$ and $2 \geq p>1$, then

$$
\left\|\sup _{s \in(0, R)}\left|\left(R_{a, z} \mu_{s}\right) * f\right|\right\|_{p} \leq C_{z}\|f\|_{p} \quad \forall f \in C(G) .
$$

Interpolating between these two results, using Stein's complex interpolation method [St1], yields the theorem. 
Now we prove (9). We fix $b$, greater than $\gamma+1$, and $w$ in $\left[-\frac{3}{4},-\frac{1}{2}\right]$ (so that (4) holds), sufficiently close to $-\frac{1}{2}$ that $\operatorname{Re}(w)+\operatorname{Re}(z)+\gamma>0$. We also choose $\varepsilon$ in $\mathbf{R}^{+}$such that $\operatorname{Re}(w)+\operatorname{Re}(z)+\gamma>\varepsilon$, and apply Lemma 2 .

For simplicity of notation, we write $\phi_{s}$ for $R_{a, z} \mu_{s}$ and $h_{s}$ for

$$
\frac{\Gamma(a / 2) \Gamma(b / 2)}{\Gamma(a / 2+z) \Gamma(b / 2+w)} R_{2,1} \mu_{s} .
$$

By Lemma 2, for all $s$ in $(0, R)$ and all $\lambda$ in $\Lambda$,

$$
\begin{aligned}
\mid\left(R_{b, w}\right. & \left.R_{a, z} \mu_{s}-h_{s}\right)^{\wedge}(\lambda) \mid \\
\leq & \left|\left(R_{b, w} R_{a, z} \mu_{s}\right)^{\wedge}(\lambda)-\frac{\Gamma(a / 2) \Gamma(b / 2)}{\Gamma(a / 2+z) \Gamma(b / 2+w)}\right| \\
& +\left|\frac{\Gamma(a / 2) \Gamma(b / 2)}{\Gamma(a / 2+z) \Gamma(b / 2+w)}\right|\left|\left(R_{2,1} \mu_{s}\right)^{-}(\lambda)-1\right| \\
\leq & C_{z} s|\lambda|,
\end{aligned}
$$

where $C_{z}$ grows at most exponentially in $\operatorname{Im}(z)$. Further, there is a small positive $\delta$ such that

$$
\begin{aligned}
\left|\left(R_{b, s} R_{a, z} \mu_{s}-h_{s}\right)^{-}(\lambda)\right| & \leq\left|\left(R_{b, w} R_{a, z} \mu_{s}\right)^{-}(\lambda)\right|+\left|\hat{h}_{s}(\lambda)\right| \\
& \leq C_{z}(1+s|\lambda|)^{-\delta}+C(1+s|\lambda|)^{-\gamma} \\
& \leq C_{z}(1+s|\lambda|)^{-\delta}
\end{aligned}
$$

for all $s$ in $(0, R)$ and all $\lambda$ in $\Lambda$, also by Lemma 2. From (4) above,

$$
\begin{aligned}
\left|\phi_{s} * f(x)\right|= & \left|C_{b, w} \int_{0}^{1} t^{b-1+2 w}\left(1-t^{2}\right)^{-w-1} R_{b, w} R_{a, z} \mu_{s t} * f(x) d t\right| \\
\leq & C_{b, w}\left\{\int_{0}^{1} t^{2 b+4 w-2}\left(1-t^{2}\right)^{-2 w-2} d t\right\}^{1 / 2} \\
& \cdot\left\{\int_{0}^{1}\left|R_{b, w} R_{a, z} \mu_{s t} * f(x)\right|^{2} d t\right\}^{1 / 2} \\
\leq & C_{b, w}\left\{\frac{1}{s} \int_{0}^{s}\left|R_{b, w} R_{a, z} \mu_{s t} * f(x)\right|^{2} d t\right\}^{1 / 2} \\
\leq & C_{b, w}\left\{\frac{1}{s} \int_{0}^{s}\left|\left(R_{b, w} R_{a, z} \mu_{t}-h_{t}\right) * f(x)\right|^{2} d t\right\}^{1 / 2} \\
& +C_{b, w}\left\{\frac{1}{s} \int_{0}^{s}\left|h_{t} * f(x)\right|^{2} d t\right\}^{1 / 2} \\
\leq & C_{b, w}\left\{\int_{0}^{R}\left|\left(R_{b, w} R_{a, z} \mu_{t}-h_{t}\right) * f(x)\right|^{2} \frac{d t}{t}\right\}^{1 / 2} \\
& +C_{b, w} \sup _{s \in(0, R)}\left|h_{s} * f(x)\right| \\
= & C_{b, w} g(f)(x)+C_{b, w} \sup _{s \in(0, R)}\left|h_{s} * f(x)\right| .
\end{aligned}
$$


To finish off the proof of (9), observe that this last expression is independent of $s$; it now suffices to show that $\|g(f)\|_{2} \leq C\|f\|_{2}$, since $\left\|\sup _{s \in(0, R)}\left|h_{s} * f\right|\right\|_{2} \leq$ $C\left\|_{1} f\right\|_{2}$ by Lemma 3 .

By Plancherel's and Fubini's theorems,

$$
\begin{aligned}
\|g(f)\|_{2}^{2} & =\int_{G} \int_{0}^{R}\left|\left(R_{b, w} R_{a, z} \mu_{t}-h_{t}\right) * f(x)\right|^{2} \frac{d t}{t} d g \\
& =\int_{0}^{R} \sum_{\lambda} d_{\lambda}\left|\left(R_{b, w} R_{a, z} \mu_{t}-h_{t}\right)^{-}(\lambda)\right|^{2}\left\|\pi_{\lambda}(f)\right\|_{H S}^{2} \frac{d t}{t} \\
& =\sum_{\lambda \neq 0} d_{\lambda}\left\|\pi_{\lambda}(f)\right\|_{H S}^{2} \int_{0}^{R}\left|\left(R_{b, w} R_{a, z} \mu_{t}-h_{t}\right)^{-}(\lambda)\right|^{2} \frac{d t}{t} .
\end{aligned}
$$

We use the estimates for $\left|R_{b, w} R_{a, z} \mu_{t}-h_{t}\right|$ obtained earlier, to conclude that

$$
\|g(f)\|_{2}^{2} \leq C_{z}\|f\|_{2}^{2}
$$

as required.

\section{APPENDIX: CASE BY CASE TREATMENT OF $\gamma$}

In this section we write $\gamma(G)$ rather than $\gamma$ to emphasize the dependence of $\gamma$ on $G$. We start by observing that if $G$ is locally isomorphic to a product of simple groups $G_{1}, \ldots, G_{n}$, then

$$
\gamma(G)=\min _{1 \leq j \leq n} \gamma\left(G_{j}\right)
$$

because the roots from different simple factors are all orthogonal and do not interact. We hereafter suppose that $G$ is simple.

Recall that we are letting $\Phi^{+}$denote the set of positive roots for $(G, T)$. Now $\Phi^{+}$is irreducible and its cardinality is known. To find the constant $\gamma(G)$, we use the following device, suggested by René Beerends. For each simple root $\alpha_{J}$, take the Dynkin diagram for $\Phi^{+}$and remove the $J$ th vertex. The set of roots $\alpha$ in $\Phi^{+}$which have $c_{J}(\alpha)=0$ are the positive roots of the system determined by the resulting diagram, which in general will be a union of Dynkin diagrams. Since the number of positive roots coming from a given Dynkin diagram can be calculated, we can make a list of the possible values of $\gamma(G)$ in a case by case argument.

The number of positive roots associated to the various simple root systems can be read off from pages $250-275$ in [Bo]. The resulting values for $\gamma(G)$ are tabulated here.

$\begin{array}{cccccc}\text { Root system } & \left|\Phi^{+}\right| & \gamma(G) & \text { Root system } & \left|\Phi^{+}\right| & \gamma(G) \\ A_{l}(l \geq 1) & l(l+1) / 2 & l & B_{l}(l \geq 2) & l^{2} & 2 l-1 \\ C_{l}(l \geq 3) & l^{2} & 2 l-1 & D_{l}(l \geq 4) & l(l-1) & 2 l-2 \\ G_{2} & 6 & 5 & F_{4} & 24 & 15 \\ E_{6} & 36 & 16 & E_{7} & 63 & 27 \\ E_{8} & 120 & 57 & & & \end{array}$




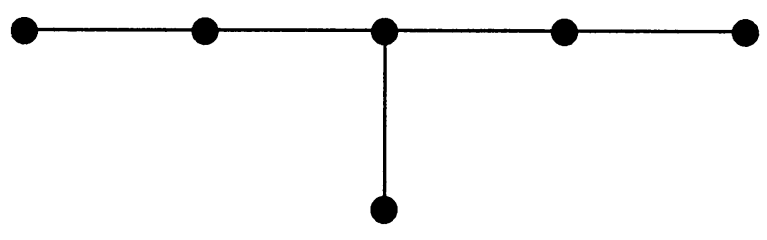

The Dynkin diagram for $E_{6}$

As an illustration of this, consider the case when $\Phi^{+}$is of type $E_{6}$.

The diagrams which result from removing one vertex are $D_{5}, A_{1} \cup A_{4}, A_{2} \cup$ $A_{1} \cup A_{2}$, and $A_{5}$. The corresponding numbers of positive roots are $20,1+$ $10=11,3+1+3=7$, and 15 . The number of positive roots in $E_{6}$ is 36 . Hence $\gamma\left(E_{6}\right)=16=\min \{16,25,29,21\}$.

\section{REFERENCES}

[Bo] N. Bourbaki, Groupes et algèbres de Lie, Chapitres 4, 5 et 6, Hermann, Paris, 1968.

[Ch] M. Christ, Averages of functions over submanifolds, Preprint, February 1988.

[CW] R. R. Coifman and G. Weiss, Transference methods in analysis, CBMS Regional Conf. Ser. Math., No. 31, Amer. Math. Soc., Providence, R.I., 1976.

[CM] M. Cowling and G. Mauceri, Inequalities for some maximal functions. II. Trans. Amer. Math. Soc. 296 (1986), 341-365.

[SS] C. D. Sogge and E. M. Stein, Averages over hypersurfaces. III-Smoothness of generalized Radon transforms, Preprint.

[St1] E. M. Stein, Interpolation of linear operators, Trans. Amer. Math. Soc. 83 (1956), 482-492.

[St2] __, Maximal functions: spherical means, Proc. Nat. Acad. Sci. U.S.A. 73 (1976), 2174-2175.

[Wa] N. R. Wallach, Harmonic analysis on homogeneous spaces, Dekker, New York, 1973.

School of Mathematics, University of New South Wales, Kensington, N.S.W. 2033, AUSTRALIA

Department of Mathematics, ias, Australian National University, Canberra, ACT 2601, Australia 